INDO GLOBAL JOURNAL OF

PHARMACEUTICAL SCIENCES

ISSN 2249- 1023

\title{
Investigating the Role of PGPM in Assisting Plant Growth Under Stress Caused by Organophosphate Pesticide- Phorate
}

\author{
Sukriti Gupta, Srishti Dangayach, Kriti Shukla, Nikhita Agrawal, S Krishna Sundari * \\ Biotechnology Department, Jaypee Institute of Information Technology (JIIT), A-10, Sector-62, Noida-201307, India
}

Address for Correspondence: S Krishna Sundari; krishna.sundari@jiit.ac.in, sukritigupta123@gmail.com

\begin{abstract}
Farmers across the world face the challenge of compromised agriculture productivity due to abiotic stress. Residual pesticides accumulating in the soil rhizospere are often found to cause a chemical imbalance in the soil microclimate and cause chemical stress and toxicity. Plant growth promoting rhizobacteria (PGPR) are popularly studied for their abilities to enhance plant growth by providing stress tolerance to plants. The present study is to investigate the role of PGPR in reducing toxicity caused due to accumulation of organophosphate pesticide (OPP) - Phorate. We were successful in isolating, evaluating and selecting eleven PGPR strains by various rounds of purification and their response to OPP by performing biochemical assay. Isolates of PGPR from the rhizospere of Bajara-PB, Makka-PM, Arhar-PA and Sugarcane-PS were studied for their ability to tolerate a wide range of OPP (75 ppm to $600 \mathrm{ppm})$. When compared with control, OPP stress subjected PA showed best tolerance to OPP with a percentage increment in growth of $20.1 \%$ at 380ppm of OPP. Subsequently, in vitro plant bioassay was performed to check ability of PGPR for promoting growth of Mung beans (Vigna radiata) when subjected to OPP concentration as stated above. The parameters studied were percentage germination, radical and plumule length, leaf count, root:shoot ratio, seed vigour and total biomass. Upon inoculation with PGPR, positive growth in radical length was observed but no substantial increase in plumule length was seen in Mung beans seedlings. Also, there was increase in percentage germination, leaf count, seed vigour and total biomass in seedlings with PGPR. This study clearly indicates that inoculation with PGPR can have a positive impact on plant growth even under high concentration of organophosphate pesticides. (C) 2014 iGlobal Research and Publishing Foundation. All rights reserved.
\end{abstract}

Conference Proceedings: International Conference on Life Sciences, Informatics, Food and Environment; August 2930, 2014

Indo Global Journal of Pharmaceutical Sciences( ISSN 22491023 ; CODEN-I IGJPAI; NLM ID: 101610675) indexed and abstracted in EMBASE(Elsevier), SCIRUS(Elsevier),CABI, CAB Abstracts, Chemical Abstract Services(CAS), American Chemical Society(ACS), Index Copernicus, EBSCO, DOAJ, Google Scholar and many more. For further details, visit 